Document downloaded from:

http://hdl.handle.net/10251/66699

This paper must be cited as:

De Pereda Sebastián, D.; Romero Vivó, S.; Ricarte Benedito, B.; Rossetti, P.; Ampudia Blasco, FJ.; Bondía Company, J. (2015). Real-time estimation of plasma insulin concentration from continuous glucose monitor measurements. Computer Methods in Biomechanics and Biomedical Engineering. Sep:1-9. doi:10.1080/10255842.2015.1077234

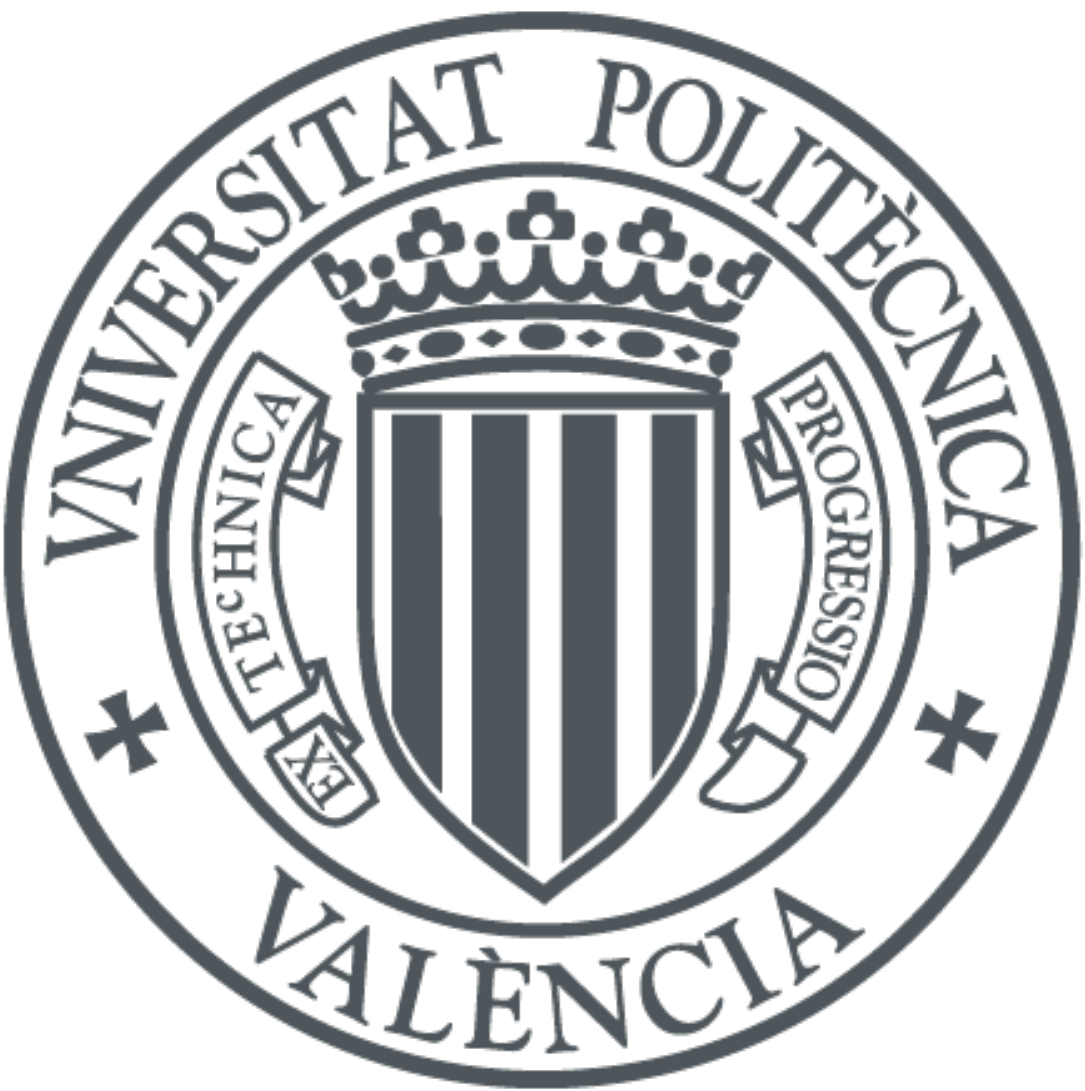

The final publication is available at

http://dx.doi.org/10.1080/10255842.2015.1077234

Copyright Taylor \& Francis

Additional Information 


\title{
Real-time estimation of plasma insulin concentration from continuous glucose monitor measurements
}

\author{
Diego de Pereda ${ }^{\mathrm{a}}$, Sergio Romero-Vivo ${ }^{\mathrm{b}}$, Beatriz Ricarte ${ }^{\mathrm{b}}$, Paolo Rossetti ${ }^{\mathrm{c}}$, Francisco Javier \\ Ampudia-Blasco ${ }^{\mathrm{d}}$ and Jorge Bondia ${ }^{\mathrm{a}, *}$ \\ ${ }^{a}$ Institut Universitari d'Automàtica i Informàtica Industrial, Universitat Politècnica de València, Spain \\ ${ }^{b}$ Institut Universitari de Matemàtica Multidisciplinar, Universitat Politècnica de València, Spain \\ ${ }^{c}$ Departamento de Medicina Interna, Hospital Sant Francesc de Borja, Gandía, Valencia, Spain \\ ${ }^{d}$ Diabetes Reference Unit, Endocrinology and Nutrition Department, Valencia Clinic University Hospital, \\ Spain
}

\begin{abstract}
Continuous glucose monitors can measure interstitial glucose concentration in real-time for closed-loop glucose control systems, known as artificial pancreas. These control systems use an insulin feedback to maintain plasma glucose concentration within a narrow and safe range, and thus to avoid health complications. As it is not possible to measure plasma insulin concentration in real-time, insulin models have been used in literature to estimate them. Nevertheless, the significant inter- and intra-patient variability of insulin absorption jeopardizes the accuracy of these estimations. In order to reduce these limitations, our objective is to perform a real-time estimation of plasma insulin concentration from continuous glucose monitoring.

Hovorka's glucose-insulin model has been incorporated in an Extended Kalman Filter in which different selected time-variant model parameters have been considered as extended states. The observability of the original Hovorka's model and of several extended models has been evaluated by their Lie derivatives. We have evaluated this methodology with an in-silico study with 100 patients with Type 1 diabetes during 25 hours. Furthermore, it has been also validated using clinical data from 12 insulin pump patients with Type 1 diabetes who underwent four mixed meal studies. Real-time insulin estimations have been compared to plasma insulin measurements to assess performance showing the validity of the methodology here used in comparison with that formerly used for insulin models. Hence, real-time estimations for plasma insulin concentration based on subcutaneous glucose monitoring can be beneficial for increasing the efficiency of control algorithms for the artificial pancreas.
\end{abstract}

Keywords: Extended Kalman filter; Insulin estimation; Glucose-insulin models; Type 1 diabetes; Artificial Pancreas

\section{Introduction}

Under physiological conditions plasma glucose is maintained in a narrow range $(70-140 \mathrm{mg} / \mathrm{dl})$. Insulin, a peptide secreted from the pancreatic $\beta$-cells, is the main actor of glucose homeostasis. In the fasting and post-absorptive states, insulin regulates plasma glucose concentration mainly restraining hepatic glucose production to exactly match peripheral glucose utilization (predominantly by the brain, muscle and adipose tissue). In the post-prandial state, the increase in the portal glucose concentration stimulates insulin secretion, which in turn suppresses hepatic glucose production and increases the disposal of the glucose absorbed from the gut (Gerich 2000).

Patients affected by Type 1 diabetes suffer from an absolute insulin deficiency, due to the autoimmune destruction of the pancreatic $\beta$-cells. Before the discovery of insulin in 1929, this led to fatal acute complications (hyperglycemia, ketoacidosis, coma and death). Following 1929, thanks to the

*Corresponding author. Tel.: +34 963877007; Fax: +34 963879579; Email: jbondia@isa.upv.es 
development of progressively more sophisticated insulin formulations and devices for glucose measurements, acute diabetic complications are rare (although not negligible). However, physiological replacement of insulin secretion remains an elusive goal. This fact results into wide fluctuations of plasma glucose (including life-threatening hypoglycemia) and chronic hyperglycemia, the latter being the cause of significant morbidity (end-stage renal disease, blindness, neuropathy) and mortality.

For the above reasons, a significant amount of research has been focused on the development of an artificial pancreas, i.e., a closed-loop glucose control system that automatically dispenses insulin subcutaneously. The investigation in this field has been fuelled in the last decade following the introduction in the market of portable devices for real-time continuous glucose monitoring (CGM) (Rossetti, Bondia, et al. 2010). However, one of the main limitations to a physiological insulin replacement remains the estimation of plasma insulin concentration. Since insulin cannot be measured in real-time, insulin models have been used to estimate such concentration (Patek, Magni, et al. 2012; Swan, Dziura, et al. 2009). However, variability of pharmacokinetic dynamics jeopardizes the accuracy of the estimation (de Pereda, Romero-Vivo, et al. 2012). This variability is due to the distribution of the injection and the characteristics of the subcutaneous tissue, and it is basically focused on two parameters: time-to-peak plasma insulin concentration and metabolic clearance rate of insulin (Haidar, Elleri, et al. 2013; Radziuk and Lickley 1985). This question limits the performance of any artificial pancreas potentially, leading to either over- or under-insulinization and posing patient's safety at risk. Effective closed-loop glucose control requires the design of robust strategies integrating patient's variability into tools for an accurate plasma insulin estimation.

The limited set of measurements can be overcome with the conjunction of a model and a state observer (Dochain 2003). Observers like the Kalman Filter (Dochain 2003; Kalman, et al. 1960) can be used in continuous- or discrete-time, or combining discrete measurements in continuous-time (Crassidis and Junkins 2011). Kalman Filters have been used to enhance the accuracy of continuous glucose monitors (Facchinetti, Sparacino and Cobelli 2010; Knobbe and Buckingham 2005) in humans. Furthermore, they have been used to estimate insulin concentration from intravenous glucose tolerance tests (IVGTT) in non-diabetic humans (Eberle and Ament 2011), and from subcutaneous (SC) glucose measurements in diabetic and non-diabetic pigs (Eberle and Ament 2012). We advance one step further, as in this paper, we have tackled the problem of estimating in real-time plasma insulin concentration for diabetic human patients. This study is much closer to the artificial pancreas real performance than previous studies, as it is applied to diabetic human patients in postprandial state (not IVGTTs) with glucose measurements from a SC glucose monitor. We have evaluated different approaches in an in-silico study with 100 Type 1 diabetes patients during 25 hours, and using clinical data from 12 insulin pump patients with Type 1 diabetes who underwent four mixed meal studies (Rossetti, Ampudia-Blasco, et al. 2012).

The paper has been organised as follows. In Section 2, several approaches for a real-time estimation of plasma insulin concentration from SC continuous glucose monitoring are introduced. In Section 3, insulin estimations are validated in both an in-silico study and using real data from diabetic patients. In Section 4, observability of the systems is reviewed, and real-time insulin estimations are compared to plasma insulin measurements to assess performance. Finally, Section 5 includes a discussion and outlines the conclusions of this study.

\section{Real-Time Estimation of Plasma Insulin Concentration}

In this section, several approaches for an estimation of plasma insulin concentration in real-time are presented. A specific model has been chosen to illustrate these techniques, although they could be easily extended to any other model. 
Table 1. Set of differential equations for the Hovorka's glucose-insulin model.

\begin{tabular}{|c|c|c|c|}
\hline State & Differential equation & & Parameters and inputs \\
\hline $\begin{array}{l}\text { Subcutaneous insulin absorption } \\
{[m U]}\end{array}$ & $\begin{array}{l}\dot{S}_{1}(t)=u(t)-\frac{S_{1}(t)}{t_{\max , I}} \\
\dot{S}_{2}(t)=\frac{S_{1}(t)}{t_{\max , I}}-\frac{S_{2}(t)}{t_{\max , I}}\end{array}$ & $\begin{array}{c}u(t) \\
t_{\max , I}\end{array}$ & $\begin{array}{l}\text { Administration of basal and bolus insulin }[\mathrm{mU} / \mathrm{min}] \\
\text { Time-to-maximum insulin absorption }[=55 \mathrm{~min}]\end{array}$ \\
\hline $\begin{array}{l}\text { Plasma insulin concentration } \\
{[m U / L]}\end{array}$ & $\dot{I}(t)=\frac{S_{2}(t)}{t_{\max , I} V_{I}}-k_{e} I(t)$ & $\begin{array}{l}V_{I} \\
k_{e}\end{array}$ & $\begin{array}{l}\text { Insulin distribution volume }[=0.12 *(\text { Patient weight }) L] \\
\text { Fractional elimination rate }\left[=0.138 \mathrm{~min}^{-1}\right]\end{array}$ \\
\hline $\begin{array}{l}\text { Effects of insulin on the distribution } \\
\text { and transport of glucose }\left[\mathrm{min}^{-1}\right]\end{array}$ & $\dot{x}_{1}(t)=-k_{a 1} x_{1}(t)+k_{b 1} I(t)$ & $\begin{array}{l}k_{a 1} \\
k_{b 1}\end{array}$ & $\begin{array}{l}\text { Deactivation rate constant }\left[=0.006 \mathrm{~min}^{-1}\right] \\
\text { Activation rate constant }\left[=k_{a 1} * 51.2 * 10^{-4} \mathrm{~min}^{-1}\right]\end{array}$ \\
\hline $\begin{array}{l}\text { Effect of insulin on the glucose } \\
\text { disposal }\left[\mathrm{min}^{-1}\right]\end{array}$ & $\dot{x}_{2}(t)=-k_{a 2} x_{2}(t)+k_{b 2} I(t)$ & $\begin{array}{l}k_{a 2} \\
k_{b 2}\end{array}$ & $\begin{array}{l}\text { Deactivation rate constant }\left[=0.06 \mathrm{~min}^{-1}\right] \\
\text { Activation rate constant }\left[=k_{a 2} * 8.2 * 10^{-4} \mathrm{~min}^{-1}\right]\end{array}$ \\
\hline $\begin{array}{l}\text { Effect of insulin on the production } \\
\text { of endogenous glucose }[\text { Unitless] }\end{array}$ & $\dot{x}_{3}(t)=-k_{a 3} x_{3}(t)+k_{b 3} I(t)$ & $\begin{array}{l}k_{a 3} \\
k_{b 3}\end{array}$ & $\begin{array}{l}\text { Deactivation rate constant }\left[=0.03 \mathrm{~min}^{-1}\right] \\
\text { Activation rate constant }\left[=k_{a 3} * 520 * 10^{-4} \mathrm{~min}^{-1}\right]\end{array}$ \\
\hline $\begin{array}{l}\text { Carbohydrate absorption rate } \\
{[\mathrm{mmol} / \mathrm{min}]}\end{array}$ & $U_{G}(t)=\frac{D_{G} A_{G} t e^{-t / t_{\max , G}}}{t_{\max , G}^{2}}$ & $\begin{array}{c}D_{G} \\
A_{G} \\
t_{\max , G}\end{array}$ & $\begin{array}{l}\text { Amount of carbohydrates digested }[\mathrm{mmol}] \\
\text { Carbohydrate bioavailability }[=0.8(\text { Unitless })] \\
\text { Time-of-maximum appearance of glucose in the } \\
\text { accessible compartment }[=40 \mathrm{~min}]\end{array}$ \\
\hline $\begin{array}{l}\text { Glucose masses in the accessible } \\
\text { compartment }[\mathrm{mmol}]\end{array}$ & $\begin{array}{l}\dot{Q}_{1}(t)=-x_{1}(t) Q_{1}(t) \\
+k_{12} Q_{2}(t)-F_{1}^{c}(t)-F_{R}(t) \\
+U_{G}(t)+E G P_{0}\left(1-x_{3}(t)\right)\end{array}$ & $\begin{array}{l}F_{01}^{c}(t) \\
F_{R}(t) \\
E G P_{0}\end{array}$ & $\begin{array}{l}\text { Non-insulin-dependent glucose disposal }[\mathrm{mmol} / \mathrm{min}] \\
\text { Renal glucose clearance }[\mathrm{mmol} / \mathrm{min}] \\
\text { Endogenous glucose production } \\
[=0.0161 * \text { Patient weight }) \mathrm{mmol} / \mathrm{min}]\end{array}$ \\
\hline $\begin{array}{l}\text { Glucose in non-accessible } \\
\text { compartment }[\mathrm{mmol}]\end{array}$ & $\begin{array}{l}\dot{Q}_{2}(t)=x_{1}(t) Q_{1}(t) \\
-k_{12} Q_{2}(t)-x_{2}(t) Q_{2}(t)\end{array}$ & $k_{12}$ & $\begin{array}{l}\text { Transfer rate from the non-accessible to the accessible } \\
\text { compartment }\left[=0.066 \mathrm{~min}^{-1}\right]\end{array}$ \\
\hline $\begin{array}{l}\text { Interstitial glucose concentration } \\
{[\mathrm{mmol} / \mathrm{L}]}\end{array}$ & $I G(t)=\frac{1}{\tau}\left(\frac{Q_{1}(t)}{V_{G}}-I G(t)\right)$ & $\begin{array}{c}V_{G} \\
\tau\end{array}$ & $\begin{array}{l}\text { Volume of the accessible compartment } \\
{[=0.16 *(\text { Patient weight }) L]} \\
\text { Time delay }[=16 \mathrm{~min}]\end{array}$ \\
\hline
\end{tabular}

\subsection{Estimation from an Insulin Model}

A common approach in literature to estimate plasma insulin concentration is to simulate the insulin intakes in an insulin model (Patek, Magni, et al. 2012; Swan, Dziura, et al. 2009). The simulation of an insulin model acts like an open-loop, as the glucose measurements are not used to adjust the states or the parameters of the system. Nevertheless, these adjustments could be necessary, especially in processes with high variability. In general, this system can be represented by:

$$
\dot{x}(t)=f(x(t), u(t))
$$

where $u(t)$ is the input of the model, $x(t)$ is the system state vector, and $f(x(t), u(t))$ defines the system's dynamics.

The insulin model considered in this work is the model developed by Hovorka et al. (Hovorka, Canonico, et al. 2004). The insulin model $f(x(t), u(t))$ is given by the first three equations of Table 1 , while the system state vector $x(t)$ is given by:

$$
x(t)=\left[S_{1}, S_{2}, I\right] .
$$




\subsection{State Observation of a Glucose-Insulin Model}

High variability processes may be represented by systems with noise in their dynamic and measurements. These systems can be expressed by:

$$
\begin{array}{ll}
\dot{x}(t)=f(x(t), u(t))+w(t), & w(t) \sim N(0, Q(t)) \\
z(t)=h(x(t))+v(t), & v(t) \sim N(0, R(t))
\end{array}
$$

where $h(x(t))$ denotes the measurement function and $z(t)$ is the output of the model. Furthermore, $w(t)$ and $v(t)$ represent the process and the observation noises, while $Q(t)$ and $R(t)$ denote the variances of these noises, respectively.

In biological systems, only a few states are measurable. In this case, only interstitial glucose concentration can be measured by SC glucose monitors. However, state observers like the Kalman filters can estimate non-measurable states based on a limited set of measurements (Dochain 2003). The Kalman filter is a stochastic filter that allows the estimation of the states of a system based on a linear state-space model. This observer estimates system states in a two-step process: the prediction and the correction steps (Dochain 2003; Kalman, et al. 1960). In the prediction step, the Kalman filter estimates the current state variables. Once a new measurement (with noise) is observed, the state estimations are updated using a weighted process.

The extended Kalman filter (EKF) uses a local linearisation to extend the scope of the Kalman filter to systems described by non-linear ordinary differential equations (Maybeck 1979; Jazwinski 2007). In order to estimate plasma insulin concentration from punctual measurements of interstitial glucose concentration, the Hovorka's glucose-insulin model (Hovorka, Canonico, et al. 2004) has been incorporated in an EKF. Moreover, an extra equation has been added to the system to describe the relationship between blood glucose and interstitial glucose measured by continuous glucose monitors (Wilinska, Bodenlenz, et al. 2004; Knobbe and Buckingham 2005). All the equations, variables, parameters, units and nominal values used in this model are introduced in Table 1 . The system state vector $x(t)$ is given by:

$$
x(t)=\left[S_{1}, S_{2}, I, x_{1}, x_{2}, x_{3}, Q_{1}, Q_{2}, I G\right]
$$

Note that $U_{G}(t)$ is not included in the state vector since it is explicitly expressed into Table 1 , and not as a dynamical equation. The function $f(x(t), u(t))$ is described by the dynamical equations exposed into Table 1, while $h(x(t))=I G(t)$, as only interstitial glucose concentration can be measured by SC glucose monitors.

The estimation of the system state vector $x(t)$ is represented by $\hat{x}(t)$ :

$$
\dot{\hat{x}}(t)=f(\hat{x}(t), u(t))+K(t)(z(t)-h(\hat{x}(t)))
$$

where $K(t)$ is the gain matrix.

The linearized transition matrix, $F$, with elements defined by $f(x(t), u(t))$, is given by:

$$
F(t)=\left.\frac{\partial f}{\partial x}\right|_{\hat{x}(t), u(t)}
$$

which the covariance matrix, $P$, forward in time, that is:

$$
\dot{P}(t)=F(t) P(t)+P(t) F(t)^{\top}-K(t) H(t) P(t)+Q(t)
$$


where $H(t)$ is the measurement matrix, given by:

$$
H(t)=\left.\frac{\partial h}{\partial x}\right|_{\hat{x}(t)}=\{0,0,0,0,0,0,0,0,1\}
$$

as only interstitial glucose $(I G)$ can be measured, i.e., $h(x(t))=I G(t)$.

Finally, the gain matrix is updated:

$$
K(t)=P(t) H(t)^{\top} R(t)^{-1}
$$

In this approach, the variances of the process and observation noises $(Q(t)$ and $R(t)$, respectively) have been heuristically adapted to to:

$$
\begin{aligned}
& Q(t)=\operatorname{diag}\left(20,20,0.01,10^{-6}, 10^{-8}, 10^{-5}, 1,1,1\right) \\
& R(t)=0.45 \mathrm{mmol} / \mathrm{L} \quad[=8 \mathrm{mg} / \mathrm{dl}] .
\end{aligned}
$$

However, it is not always possible to estimate all the system states from a limited set of measurements. Observability analysis proves if the model states in $x(t)$ can be theoretically estimated from measurements of $z(t)$. This analysis can be assessed by the Lie derivatives (Hermann and Krener 1977; Gauthier and Bornard 1981) of the glucose-insulin model. The Lie derivative of $h(x)$ with respect to $f(x, u)$ is defined as $L_{f(x, u)} h(x)=\nabla h(x) \cdot f(x, u)$, and it is calculated recursively, as follows:

$$
\begin{aligned}
& L_{f(x, u)}^{i} h(x)=L_{f(x, u)}\left(L_{f(x, u)}^{(i-1)} h(x)\right) \quad \text { if } i=1,2, \ldots \\
& L_{f(x, u)}^{0} h(x)=h(x)
\end{aligned}
$$

A function $h(x)$ is considered observable if the following Jacobian matrix

$$
\frac{\partial}{\partial x}\left[\begin{array}{c}
h(x) \\
L_{f(x, u)} h(x) \\
\cdots \\
L_{f(x, u)}^{n-1} h(x)
\end{array}\right]
$$

has rank $n$, where $n$ is the system order.

\subsection{State Observation of an Extended Model}

The main problem with simulations and state observers is that they are often developed under assuming perfect knowledge of the system dynamics, in particular of the parameter values. However, this is not true due to the high variability of the processes, which leads to uncertain parameters. State observers can be used to estimate these uncertain parameters by building new extended models in which these parameters are considered as new states with no dynamics (Dochain 2003; Eberle and Ament 2012). For this reason, extended Hovorka's models have been defined such that different parameters have been considered as extended states of the model:

$$
\frac{d}{d t}\left[\begin{array}{l}
x(t) \\
p(t)
\end{array}\right]=\left[\begin{array}{c}
\left.f(x(t), u(t))\right|_{p=p(t)} \\
0
\end{array}\right]
$$

The parameters with the greatest impact in plasma insulin concentration are $k_{e}$ (plasma insulin elimination rate) and $t_{\max , I}$ (time to maximum insulin absorption), as seen in literature (Haidar, Elleri, et al. 2013; Radziuk and Lickley 1985) and we have verified experimentaly. Hence, we are 
interested in estimating these parameters, so they have been selected as uncertain states in extended Hovorka's models, where one or both parameters have been considered as extended states. The observability of these models has been evaluated by their Lie derivatives.

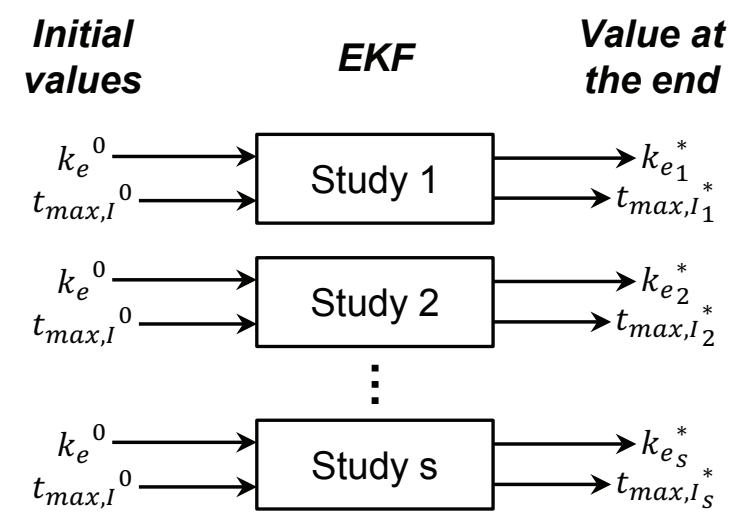

Figure 1. Diagram of the adjustment of uncertain parameters.

Then, the extended models have been incorporated in an EKF, such that the parameters $k_{e}$ and/or $t_{\max , I}$ are adapted along time, as seen in Figure 1. During each study, the system states are estimated based on punctual measurements of plasma glucose concentration. The initial value of the uncertain parameters $\left(k_{e}{ }^{0}\right.$ and $\left.t_{\max , I^{0}}\right)$ has been set at their nominal value (Hovorka, Canonico, et al. 2004) for all the studies. The value of these parameters at the end of the study is $k_{e i}^{*}$ and $t_{\max , I_{i}^{*}}, i=1, \ldots, s$, where $s$ is the number of studies. The variance of the process noise of the extended states $k_{e}$ and $t_{\max , I}$ has been added to the matrix $Q(t)$, exactly the heuristically selected values $10^{-4}$ and 1 , respectively.

\section{Validation of insulin estimations}

In this section, insulin estimations are validated in both an in-silico study and using real data from patients.

\subsection{In-silico validation}

In-silico patients with Type 1 diabetes have been simulated during 1500 minutes (25 hours), including a meal of $300 \mathrm{mmol}$ (54 grams) of glucose every 300 minutes, for a total of five meals. Five equal meals do not represent a realistic scenario, but it allows to analyse the behaviour of the estimations with varying parameters in the same scenario. Each meal is compensated with a bolus of $2300 \mathrm{mU}$ of insulin. Furthermore, each patient receives a basal insulin infusion of $10 \mathrm{mU} / \mathrm{min}$. During the simulation, the values of the parameters $k_{e}$ and $t_{\max , I}$ vary along time based on their variability. The rest of the parameters assume their nominal value.

Real-time insulin estimations of 100 in-silico patients have been computed based on interstitial glucose measurements every 15 minutes. Insulin estimations $(\hat{z})$ have been compared to plasma insulin simulation $(z(t))$ to assess the methodology introduced. The performance of the different approaches has been quantitatively evaluated by computing the Root Mean Square Error (RMSE) and the Mean Absolute Relative Deviation (MARD):

$$
\mathrm{RMSE}=\sqrt{\frac{1}{m} \sum_{t=1}^{m}\left(z_{t}-\hat{z}_{t}\right)^{2}}
$$




$$
\operatorname{MARD}=\frac{1}{m} \sum_{t=1}^{m} \frac{\left|z_{t}-\hat{z}_{t}\right|}{z_{t}}
$$

where $m$ is the number of measurements.

\subsection{Validation with real data}

State observers need several data values to adapt the system states (including the extended parameters). However, continuous glucose monitors only provide a new glucose concentration value every 1-15 minutes. Thus, the state observer is not able to adapt the system states during the first minutes of the experiment. The initial value of the system states is crucial during the first minutes of the insulin estimation, as the EKF needs some time to be able to perform an accurate estimation. If the values are under- or over-estimated, large errors may be produced in an insulin estimation. For this reason, we propose to change the initial values of the extended states, i.e. parameters $k_{e}$ and $t_{\max , I}$, for more realistic values.
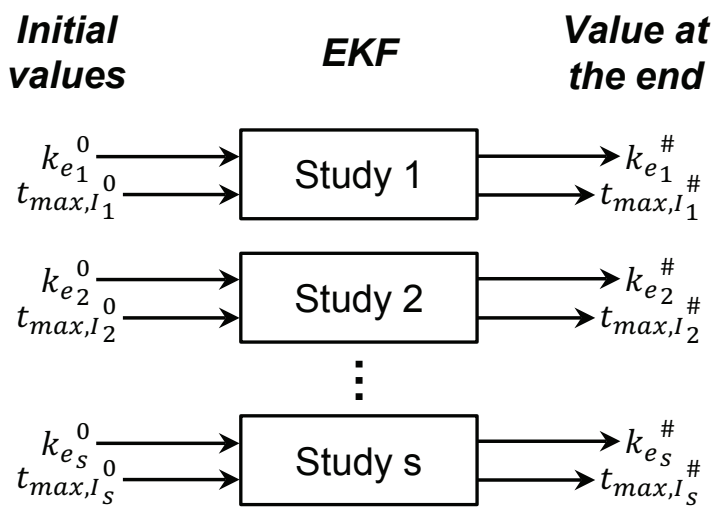

Figure 2. Diagram of the adjustment of uncertain parameters.

These new initial values are determined by a cross-validation of the estimated values of the uncertain parameters at the end of the rest of the studies:

$$
\begin{aligned}
k_{e_{i}^{0}}^{0} & =\frac{1}{s-1} \sum_{j=1}^{s} \delta_{i, j} k_{e j}^{*} \\
t_{\max , I_{i}^{0}} & =\frac{1}{s-1} \sum_{j=1}^{s} \delta_{i, j} t_{\max , I_{j}^{*}}^{*}
\end{aligned}
$$

where $s$ is the number of studies, and the function $\delta_{i, j}$ is defined as:

$$
\delta_{i, j}=\left\{\begin{array}{l}
1 \text { if } i \neq j \\
0 \text { if } i=j
\end{array}\right.
$$

These new initial values are represented in Figure 2. During each study, the value of the uncertain parameters $k_{e}$ and $t_{\max , I}$ is adapted through time.

Insulin estimations have been compared using real data from 12 insulin pump patients with Type 1 diabetes who underwent four mixed meal studies ( 9 women; $41.8 \pm 7.3$ years old; diabetes duration $20.2 \pm 10.3$ years; body mass index: $25.1 \pm 2.8 \mathrm{~kg} / \mathrm{m}^{2}$ ) (Rossetti, Ampudia-Blasco, et al. 2012). The performance of insulin estimations has been evaluated by a comparison with plasma insulin measurements, computing RMSE (2) and MARD (3). 
Table 2. Results for in-silico estimation of plasma insulin concentration applying different approaches.

\begin{tabular}{lccc}
\hline Model & RMSE $(m U / L)$ & MARD $(\%)$ & Computational Time $(\mathrm{s})$ \\
\hline Hovorka's Insulin Model & $5.6 \pm 2.9$ & $50 \% \pm 26 \%$ & $3 \cdot 10^{-6} \mathrm{~s}$ \\
Original Hovorka's EKF & $5.5 \pm 2.0$ & $51 \% \pm 26 \%$ & $0.095 \mathrm{~s}$ \\
$k_{e}$ Parameter EKF & $3.2 \pm 1.7$ & $19 \% \pm 5 \%$ & $0.098 \mathrm{~s}$ \\
$t_{\max , I}$ Parameter EKF & $3.9 \pm 1.0$ & $40 \% \pm 18 \%$ & $0.097 \mathrm{~s}$ \\
$k_{e} t_{\max , I}$ Parameters EKF & $2.4 \pm 0.6$ & $16 \% \pm 4 \%$ & $0.100 \mathrm{~s}$ \\
\hline
\end{tabular}

\section{Results}

Different estimations of plasma insulin concentration have been performed according to the approaches presented in the previous sections. All the simulations have been executed with Matlab software (version R20013a) using an Intel(R) Core i7-3770K 3.5 GHz Pentium(R) processor. The obtained results are included hereinafter and commented with detail in the discussion section.

\subsection{Observability}

The observability of the original Hovorka's model and of the extended models with the following state vectors:

$$
\begin{aligned}
& x(t)=\left\{S_{1}, S_{2}, I, x_{1}, x_{2}, x_{3}, Q_{1}, Q_{2}, I G\right\} \\
& x(t)=\left\{S_{1}, S_{2}, I, x_{1}, x_{2}, x_{3}, Q_{1}, Q_{2}, I G, k_{e}\right\} \\
& x(t)=\left\{S_{1}, S_{2}, I, x_{1}, x_{2}, x_{3}, Q_{1}, Q_{2}, I G, t_{\text {max }, I}\right\} \\
& x(t)=\left\{S_{1}, S_{2}, I, x_{1}, x_{2}, x_{3}, Q_{1}, Q_{2}, I G, k_{e}, t_{\max , I}\right\}
\end{aligned}
$$

has been evaluated by their Lie derivatives (Hermann and Krener 1977; Gauthier and Bornard 1981). In all the cases, the jacobian matrices (1) have full-rank (rank 9, 10, 10 and 11, respectively). Therefore, all the systems are observable, and it is theoretically possible to estimate all the states from $I G(t)$ interstitial glucose measurements.

\section{$4.2 \quad$ In-silico results}

In the original Hovorka's model all the model parameters are considered constant along time. However, during the in-silico simulation, parameters $k_{e}$ and $t_{\max , I}$ vary along time. These differences lead to large values for RMSE and MARD in the plasma insulin estimation, as seen in Table 2. The estimations performed through the EKF are represented with a solid line in Figure 3, while the square dots represent the values of the in-silico simulation.

The model parameters $k_{e}$ and $t_{\max , I}$ (one or both) can be considered as extended states in the Hovorka's model, and incorporated in an EKF. The results obtained are represented in Table 2 , showing that the best results are achieved when both parameters are considered as extended states. This estimation is shown with a dotted line in Figure 3. This approach improves significantly $(p<0.001)$ the results obtained with the simulation of Hovorka's insulin model. The computational time for each approach is shown in Table 2 .

\subsection{Results with real data}

The simulation of Hovorka's insulin model overestimates the plasma insulin concentration in all the patients. Almost the same overestimation is obtained when the estimation of all the system states is performed through an EKF, represented with a solid line in Figure 4, while the square dots represent real measurements of plasma insulin and glucose concentration. The plasma insulin overestimation leads to large values for RMSE and MARD, as shown in Table 3. 

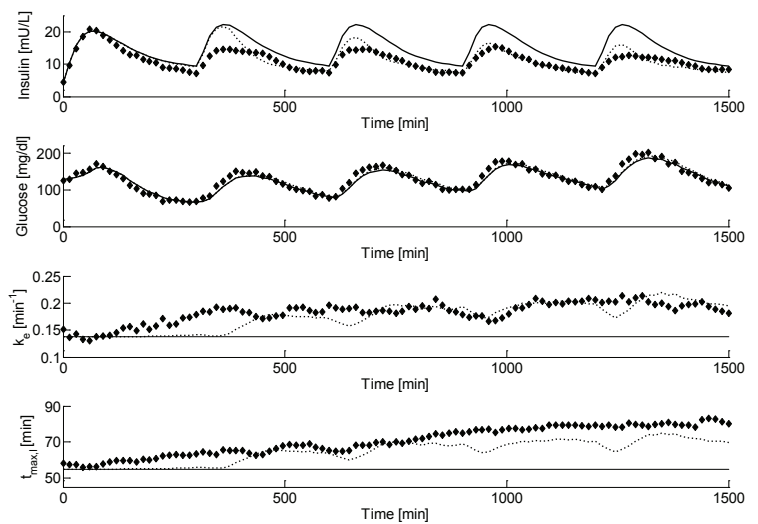

Figure 3. Example of in-silico estimations of plasma insulin concentration. The square dots represent measurements from the in-silico simulation, while the lines represent several estimation approaches: Original Hovorka EKF (solid line) and $k_{e}-t_{m a x, I}$ Parameters EKF (dotted line).
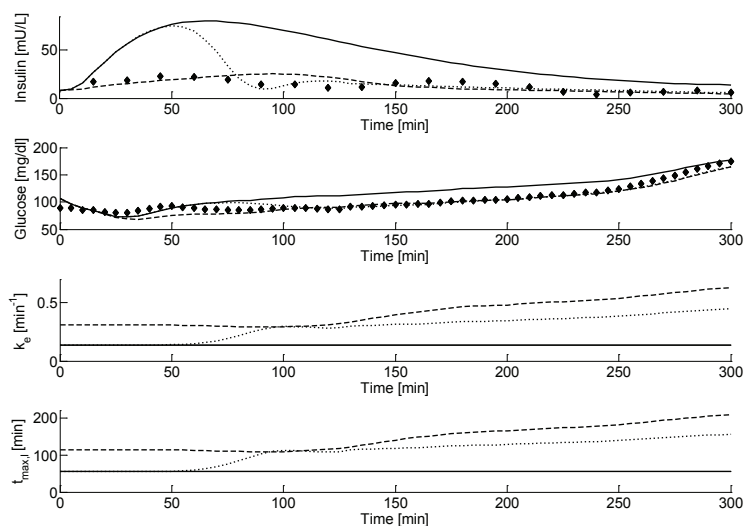

Figure 4. Example of estimations of plasma insulin concentration with real data. The square dots represent real measurements, while the lines represent several estimation approaches: Original Hovorka EKF (solid line), $k_{e}-t_{m a x, I}$ Parameters EKF (dotted line) and Cross-Validation $k_{e}-t_{\max , I}$ EKF (dashed line).

Table 3. Results for estimation of plasma insulin concentration applying different approaches with real data.

\begin{tabular}{lccc}
\hline Model & RMSE $(m U / L)$ & MARD $(\%)$ & Computational Time (s) \\
\hline Hovorka's Insulin Model & $24.5 \pm 16.5$ & $179 \% \pm 158 \%$ & $3 \cdot 10^{-6} \mathrm{~s}$ \\
Original Hovorka's EKF & $24.4 \pm 16.4$ & $177 \% \pm 156 \%$ & $0.095 \mathrm{~s}$ \\
$k_{e}$ Parameter EKF & $15.3 \pm 7.6$ & $87 \% \pm 90 \%$ & $0.098 \mathrm{~s}$ \\
$t_{\max , I}$ Parameter EKF & $18.7 \pm 11.1$ & $133 \% \pm 134 \%$ & $0.097 \mathrm{~s}$ \\
$k_{e}-t_{\max , I}$ Parameters EKF & $14.9 \pm 7.7$ & $85 \% \pm 100 \%$ & $0.100 \mathrm{~s}$ \\
Cross-Validation $k_{e}-t_{\max , I}$ EKF & $6.6 \pm 3.9$ & $49 \% \pm 72 \%$ & $0.100 \mathrm{~s}$ \\
\hline
\end{tabular}

In the above estimations, the parameters of Hovorka's model have been considered constant along time. However, the model parameters $k_{e}$ and $t_{\max , I}$ can be adapted if they are considered as extended states in the Hovorka's model. Each extended model has been incorporated in an EKF. The best results are obtained when both parameters are considered as extended states, as seen in Table 3. These results are represented in Figure 4 with a dotted line. This approach improves significantly $(p<0.001)$ the results obtained with the simulation of Hovorka's insulin model.

Finally, the estimations further improve after a cross-validation of the estimated values at the end of the studies of the uncertain parameters $k_{e}\left(0.32 \pm 0.10 \mathrm{~min}^{-1}\right)$ and $t_{\max , I}(115 \pm 33 \mathrm{~min})$, as shown in Table 3 and in Figure 4 with a dashed line. The computational time for each approach is shown in Table 3. 
Table 4. Results for real-time estimation of plasma insulin concentration applying different approaches (excluding the outlier patient).

\begin{tabular}{lccc}
\hline Model & RMSE $(m U / L)$ & MARD $(\%)$ & Computational Time $(\mathrm{s})$ \\
\hline Hovorka's Insulin Model & $23.5 \pm 14.9$ & $145 \% \pm 103 \%$ & $3 \cdot 10^{-6} \mathrm{~s}$ \\
Original Hovorka's EKF & $23.4 \pm 14.9$ & $141 \% \pm 101 \%$ & $0.095 \mathrm{~s}$ \\
$k_{e}$ Parameter EKF & $14.7 \pm 6.8$ & $66 \% \pm 25 \%$ & $0.098 \mathrm{~s}$ \\
$t_{\max , I}$ Parameter EKF & $17.8 \pm 9.9$ & $101 \% \pm 66 \%$ & $0.097 \mathrm{~s}$ \\
$k_{e}-t_{\max , I}$ Parameters EKF & $14.3 \pm 6.9$ & $61 \% \pm 28 \%$ & $0.100 \mathrm{~s}$ \\
Cross-Validation $k_{e}-t_{\max , I} \mathrm{EKF}$ & $6.4 \pm 3.9$ & $33 \% \pm 14 \%$ & $0.100 \mathrm{~s}$ \\
\hline
\end{tabular}

\section{Discussion and Conclusion}

The experiments carried out in the previous section illustrate several approaches to estimate plasma insulin concentration through SC interstitial glucose measurements in Hovorka's model. This model has been chosen to illustrate the techniques, although they could be extended to other models.

In both the in-silico and the real data studies, a large error is commited when the Hovorka's insulin model is used (a common approach in literature), as seen in Tables 2 and 3. In this model, a perfect knowledge of the system parameters is assumed, as they remain constant along time. The insulin estimation is significantly improved when parameters $k_{e}$ and $t_{\max , I}$ are considered as extended states. Once the parameters are adjusted, plasma insulin estimations are also adjusted. Consequently, time variations in both parameters may be responsible, at least in part, of intraand inter-patient variability in insulin stacking, leading to inaccurate plasma insulin estimations. When these parameters are considered as extended states, it is possible to perform individualized estimations of plasma insulin concentration.

However, the EKF need several glucose measurements to adapt the system parameters. In most of real-data cases, the nominal values of parameters $k_{e}$ and $t_{\max , I}$ (Hovorka, Canonico, et al. 2004) are underestimated, producing a large overestimation of plasma insulin concentration. After the cross-validation, the errors decrease (Table 3), specially those during the first minutes of the estimation (Figure 4). When this final approach is applied, both RMS and MARD errors are reduced in a $73 \%$ with respect to the simulation of Hovorka's insulin model. Therefore, we propose to apply this approach for the estimation of plasma insulin concentration through interstitial glucose measurements.

Observing Figure 4, it may seem that once the parameters are adapted, they do not vary much along time. However, these parameters may vary significantly during long-term estimations. In Figure 3 , the initial values of parameters $k_{e}$ and $t_{\max , I}$ are similar to the one used in the estimations. Both the Original Hovorka's EKF and the $k_{e}-t_{\max , I}$ EKF accomplish an accurate estimation of insulin concentration during the first meal. However, once the parameters values have varied, only the $k_{e}-t_{\max , I} \mathrm{EKF}$ is able to adapt itself and perform much better estimations of plasma insulin concentration than the Original Hovorka's EKF. Hence, in long-term estimations it is important to consider $k_{e}$ and $t_{\max , I}$ parameters as extended states to adapt them along time.

Nevertheless, there is still a significant error, but it is not totally inevitable. This is, in part, a consequence of the error committed in the interstitial glucose measurements, as the SC continuous glucose monitors do not accomplish accurate measurements (Barceló-Rico, Bondia, et al. 2012). Errors in plasma insulin estimations may decrease if continuous glucose monitors improve their performance. Furthermore, in exception of $k_{e}$ and $t_{\max , I}$, the rest of the parameters of Hovorka's model assume their nominal value during the estimations. However, some of these parameters, such as the insulin sensitivity, have large variability. An individualization of these parameter values may improve plasma insulin estimations.

Most of the error committed (Table 3 ) is accumulated in a single outlier patient, in which insulin measurements do not represent reliable values. If this outlier patient is not considered in the calculations, the metrics improve significantly, specially the MARD value (Table 4).

The aim of this work was to estimate plasma insulin concentration from subcutaneous glucose measurements. Previous studies have carried out insulin estimations in non-diabetic humans after 
intravenous glucose injections (Eberle and Ament 2011), and in diabetic and non-diabetic pigs (Eberle and Ament 2012). In this work, we have analysed the behaviour of diabetic human patients after a meal ingestion, based on SC glucose measurements. In conclusion, this study approaches us to the real operation of the artificial pancreas, and improves the performance of real-time estimations for plasma insulin concentration, with respect to insulin models used in literature.

From these results based on SC glucose monitoring, we consider that the proposed approach may be beneficial for increasing the efficiency and safety of control algorithms for the artificial pancreas.

\section{Funding}

This work was partially supported by the Spanish Ministerio de Ciencia e Innovación through Grant DPI-2010-20764-C02-01 and Grant DPI-2013-46982-C2-1-R, and the European Union through FEDER fund.

\section{References}

Barceló-Rico F, Bondia J, Díez JL, Rossetti P. 2012. A multiple local models approach to accuracy improvement in continuous glucose monitoring. Diabetes technology \& therapeutics. 14(1):74-82.

Crassidis JL, Junkins JL. 2011. Optimal estimation of dynamic systems. CRC press.

de Pereda D, Romero-Vivo S, Ricarte B, Bondia J. 2012. On the prediction of glucose concentration under intra-patient variability in type 1 diabetes: A monotone systems approach. Computer Methods and Programs in Biomedicine. 108(3):993 - 1001.

Dochain D. 2003. State and parameter estimation in chemical and biochemical processes: a tutorial. Journal of process control. 13(8):801-818.

Eberle C, Ament C. 2011. The unscented kalman filter estimates the plasma insulin from glucose measurement. Biosystems. 103(1):67-72.

Eberle C, Ament C. 2012. Real-time state estimation and long-term model adaptation: a two-sided approach toward personalized diagnosis of glucose and insulin levels. Journal of diabetes science and technology. 6(5):1148-1158.

Facchinetti A, Sparacino G, Cobelli C. 2010. Enhanced accuracy of continuous glucose monitoring by online extended kalman filtering. Diabetes technology \& therapeutics. 12(5):353-363.

Gauthier J, Bornard G. 1981. Observability for any $\mathrm{u}(\mathrm{t})$ of a class of nonlinear systems. IEEE Transactions on Automatic Control. 26(4):922-926.

Gerich JE. 2000. Physiology of glucose homeostasis. Diabetes, Obesity and Metabolism. 2(6):345-350.

Haidar A, Elleri D, Kumareswaran K, Leelarathna L, Allen JM, Caldwell K, Murphy HR, Wilinska ME, Acerini CL, Evans ML, et al. 2013. Pharmacokinetics of insulin aspart in pump-treated subjects with type 1 diabetes: reproducibility and effect of age, weight, and duration of diabetes. Diabetes care. 36(10):e173e174.

Hermann R, Krener AJ. 1977. Nonlinear controllability and observability. IEEE Transactions on automatic control. 22(5):728-740.

Hovorka R, Canonico V, Chassin L, Haueter U, Massi-Benedetti M, Federici M, Pieber T, Schaller H, Schaupp L, Vering T, et al. 2004. Nonlinear model predictive control of glucose concentration in subjects with type 1 diabetes. Physiological measurement. 25:905.

Jazwinski AH. 2007. Stochastic processes and filtering theory. Courier Dover Publications.

Kalman RE, et al. 1960. A new approach to linear filtering and prediction problems. Journal of basic Engineering. 82(1):35-45.

Knobbe EJ, Buckingham B. 2005. The extended kalman filter for continuous glucose monitoring. Diabetes technology \& therapeutics. 7(1):15-27.

Maybeck PS. 1979. Stochastic models, estimation, and control. Mathematics in Science and Engineering. 141(1):423.

Patek S, Magni L, Dassau E, Karvetski C, Toffanin C, De Nicolao G, Del Favero S, Breton M, Dalla Man C, Renard E, et al. 2012. Modular closed-loop control of diabetes. IEEE transactions on biomedical engineering. 59(11):2986-2999. 
Radziuk J, Lickley H. 1985. The metabolic clearance of glucose: measurement and meaning. Diabetologia. 28(6):315-322.

Rossetti P, Ampudia-Blasco FJ, Laguna A, Revert A, Vehì J, Ascaso JF, Bondia J. 2012. Evaluation of a novel continuous glucose monitoring-based method for mealtime insulin dosingthe ibolusin subjects with type 1 diabetes using continuous subcutaneous insulin infusion therapy: A randomized controlled trial. Diabetes Technology \& Therapeutics. 14(11):1043-1052.

Rossetti P, Bondia J, Vehí J, Fanelli CG. 2010. Estimating plasma glucose from interstitial glucose: the issue of calibration algorithms in commercial continuous glucose monitoring devices. Sensors. 10(12):1093610952.

Swan KL, Dziura JD, Steil GM, Voskanyan GR, Sikes KA, Steffen AT, Martin ML, Tamborlane WV, Weinzimer SA. 2009. Effect of age of infusion site and type of rapid-acting analog on pharmacodynamic parameters of insulin boluses in youth with type 1 diabetes receiving insulin pump therapy. Diabetes Care. 32(2):240-244.

Wilinska ME, Bodenlenz M, Chassin LJ, Schaller HC, Schaupp LA, Pieber TR, Hovorka R. 2004. Interstitial glucose kinetics in subjects with type 1 diabetes under physiologic conditions. Metabolism. 53(11):14841491. (14) 